\title{
Femtosecond Laser Fabricated Spike Structures for Selective Control of Cellular Behavior
}

\author{
Sabrina Schlie, ${ }^{1,2, *}$ Elena FadeEva, ${ }^{1}$ JÜrgen Koch, ${ }^{1}$ \\ ANACLET NGEZAHAYO ${ }^{2}$ AND BORIS N. CHICHKOV ${ }^{1}$ \\ ${ }^{1}$ Laser Zentrum Hannover e.V., Hollerithallee 8 \\ D-30419 Hannover, Germany \\ ${ }^{2}$ Institute of Biophysics, Leibniz University Hannover \\ Herrenhäuser Str. 2, D-30419 Hannover, Germany
}

\begin{abstract}
In this study we investigate the potential of femtosecond laser generated micrometer sized spike structures as functional surfaces for selective cell controlling. The spike dimensions as well as the average spike to spike distance can be easily tuned by varying the process parameters. Moreover, negative replications in soft materials such as silicone elastomer can be produced. This allows tailoring of wetting properties of the spike structures and their negative replicas representing a reduced surface contact area. Furthermore, we investigated material effects on cellular behavior. By comparing human fibroblasts and SH-SY5Y neuroblastoma cells we found that the influence of the material was cell specific. The cells not only changed their morphology, but also the cell growth was affected. Whereas, neuroblastoma cells proliferated at the same rate on the spike structures as on the control surfaces, the proliferation of fibroblasts was reduced by the spike structures. These effects can result from the cell specific adhesion patterns as shown in this work. These findings show a possibility to design defined surface microstructures, which could control cellular behavior in a cell specific manner.
\end{abstract}

KEY WORDS: femtosecond laser, microstructures, surface topography, water contact angle, biomedicine, cellular adhesion.

\footnotetext{
*Author to whom correspondence should be addressed.

E-mail: schlie@biophysik.uni-hannover.de
} 


\section{INTRODUCTION}

T $\mathbf{n}$ the field of biomedicine and tissue engineering the interactions 1 between cells and biomaterial surfaces are of great importance. Several efforts were undertaken to control and guide cellular behavior by introduction of changes in surface properties such as chemistry and topography in order to generate an 'intelligent' biomaterial [1,2].

Depending on the application, it would be interesting to have biomaterials, which could affect cellular behavior in a cell specific manner. Especially, the inhibition of fibroblasts is one of the goals since these cells participate to scar formation, adhere, and proliferate on the implants more quickly than other cells such as neuronal cells [3,4]. For instance, this problem occurs with cochlea implants, whose function to restore hearing in deaf patients by electrical stimulation of the auditory nerve is negatively affected by the fibroblasts [5].

The interactions between biomaterial surfaces and cells reveal that cellular effects occur in a specific order. Adhesion to surface is considered as a first messenger which stimulates intracellular signaling pathways via Ras, Rho, MAPK cascades involved in organization of cytoskeleton, proliferation, and differentiation [6-8]. Therefore, the control of cellular behavior by an 'intelligent' biomaterial could be determined by the control of adhesion to the surface - which could either be stimulated or inhibited - as morphology, proliferation, and differentiation follow as further steps.

A possible approach to control cellular adhesion would be the use of functionalized biomaterial surfaces with defined characteristics that could imitate the natural environment of the cells. In the past, many studies have concentrated on the influence of material topography in micrometer and nanometer scale. It has been demonstrated that different structures such as pores, grooves, and pits affect cell morphology, orientation, adhesion, and proliferation of cells. However, the molecular mechanisms on the basis of these effects still remains unclear and little is known about cell specific differences regarding adhesion on these structures. $[9,10]$

On the contrary, it is known that surface topography influences wettability. This finding is predicted by two established models developed by Wenzel and Cassie-Baxter [11,12]. Both models deal with the calculation of a contact angle on a structured surface, which either depends on a complete - according to Wenzel - or an incomplete according to Cassie and Baxter - wetting of the surface. Provided that chemical changes of the material are excluded, the wettability alteration is a property of the surface topography. 
This study is focused on topography-based functionalization of biomaterial surfaces for cell controlling. Surface structuring was accomplished by femtosecond laser material processing. This technique has various advantages over other methods (e.g., lithography, polymer demixing). It enables flexible and controllable surface structuring of complex geometries, and it is applicable to almost all solid materials [13]. Ultrashort pulsed laser processing has additional benefits over long-pulse laser processing: it allows a better resolution, a reduced heat-affected zone and a large variety of producible surface topographies [14-16]. For instance, laser processing of silicon surfaces in a reactive gas atmosphere enables manufacturing of self-organized quasi-periodical arrays of spikes [17]. It is very attractive to use such a topography for analysis of cell biomaterial surface interactions, because the important structure parameters (e.g., spike dimensions, spike to spike distance) can be easily controlled by tuning manufacturing parameters.

We also tested the effectiveness of negative replicas of silicon spikes fabricated in silicone elastomer in view of selective cell controlling. This replication enables a fast and reproducible texturing of soft polymer implant materials (silicone).

Moreover, we investigated the influences of microstructered surfaces on cellular behavior with focus on genotoxicity, proliferation, morphology, and adhesion. By comparing different cell types such as human fibroblasts and SH-SY5Y neuroblastoma cells, we also analyzed cell specific responses to the structures. It is demonstrated that these cell types behaved differently on the surfaces, which correlated with cell specific adhesion patterns. Therefore, these microstructures represent a promising surface topography, which is not only variable in dimensions, but which also controls cellular behavior in a cell specific manner useful for many different biomedical applications.

\section{MATERIAL AND METHODS}

\section{Fabrication of Structures}

Spike structures were fabricated by irradiation of single-crystal p-type Silicon (110) samples with femtosecond laser pulses. In our experiments we used an amplified Ti:Sapphire femtosecond laser system (Femtopower Compact Pro, Femtolasers Produktions GmbH, Austria). This system delivers linearly polarized sub-30-fs pulses at $800 \mathrm{~nm}$ and a repetition rate of $1 \mathrm{kHz}$.

The samples were placed in a vacuum chamber evacuated down to a residual pressure of $10 \mathrm{mTorr}$, und backfilled with 500 Torr $\mathrm{SF}_{6}$. 
The chamber was mounted on translation stage. A surface area of $3 \times 3 \mathrm{~mm}^{2}$ was processed by moving the sample line by line with respect to the focus. The line velocity and line-to-line distance were matched so that the average number of laser pulses per irradiated surface was 500. A computer controlled LCD element was used for laser pulse energy setting.

After laser irradiation, the samples were treated using a $10 \% \mathrm{HF}$ aqueous solution to remove oxide layer on the spikes surface. The flat silicon samples used as a reference were treated in HF as well to keep them chemically comparable to the laser processed samples.

Negative replicas of silicon spike structures were produced from two component silicone elastomer MED-4234 (NuSil Silicone Technology). The silicone elastomer was prepared according to the producer's product profile. Then it was poured over the laser fabricated spike structure, deaerated in an excicator, and finally cured at $110^{\circ} \mathrm{C}$ for $90 \mathrm{~min}$.

\section{Material Characterization}

All topographies were analyzed with a scanning electron microscope (SEM). To detect chemical changes of the samples, energy dispersive X-ray spectroscopy (EDX) was used.

Furthermore, the wettability properties of the structured samples were investigated. Here, a video-based optical contact angle measuring system (OCA 40 Micro, DataPhysics Instruments GmbH, Germany) was used. This device enables software-controlled measurement and analysis of the static and dynamic contact angles. We determined static contact angles for water using the sessile drop method. Briefly, a $2 \mu \mathrm{L}$ water drop was gently placed on the structured surface. Then, the contact angle was measured by the software system in a side view photo. All experiments were carried out at a temperature of $20^{\circ} \mathrm{C}$ and normal atmospheric conditions. Each experimental result is an average of at least five single measurements.

\section{Cell Culture}

Silicon (Si) and silicone elastomer samples were sterilized by UV light and used for cell culture experiments. Glass samples and nonstructured silicon ( $\mathrm{Si}$ ) and silicone elastomer served as references. To analyze material effects on cellular behavior human fibroblasts and SH-SY5Y neuroblastoma cells were compared. The cells were cultivated on the samples in 24 well plates filled with $2 \mathrm{~mL}$ of Dulbecco modified medium (DMEM; Sigma, Taufkirchen, Germany) supplemented with $10 \%$ fetal 
calf serum and antibiotics and were placed in a cell culture incubator (Heraeus, Hanau, Germany), in which a 95\%: $5 \%$ air: $\mathrm{CO}_{2}$ atmosphere and $80 \%$ humidity were maintained. The culture medium was renewed every $2-3$ days.

\section{Analysis of Material Genotoxicity}

In order to determine whether the laser structured materials affected the DNA of the cells, DNA strand breaking of human fibroblasts and SH-SY5Y neuroblastoma grown on spike structures and under control conditions was analyzed using the comet assay. Comet assay experiments were performed according to previous description [18]. After a cultivation time of $24 \mathrm{~h}$ the cells were trypsinized, collected, and centrifuged for $10 \mathrm{~min}$ at $800 \mathrm{~g}$. The pellets were resolved in PBS to $2 \times 10^{6}$ cells $/ \mathrm{mL}$. Later $50 \mu \mathrm{L}$ of the cell suspension was mixed with $100 \mu \mathrm{L}$ of low melting agarose $(0.6 \%)$. A $100 \mu \mathrm{L}$ of this mixture was given onto agarose-coated glass slides and covered with a cover slip. The slides were conserved for $10 \mathrm{~min}$ at $4^{\circ} \mathrm{C}$ for solidification. The cover slip was removed and further $100 \mu \mathrm{L}$ of agarose was added. After solidification at $4^{\circ} \mathrm{C}$, the slides were incubated for $90 \mathrm{~min}$ in a lysis buffer, containing $2.5 \mathrm{M} \mathrm{NaCl} ; 100 \mathrm{mM} \mathrm{Na} 2$ EDTA; $10 \mathrm{mM}$ Tris; $1 \%$ lauryl sarcosin; $1 \%$ Triton X-100; 10\% DMSO; pH 10. Subsequently, the cover slips were placed in a horizontal gel electrophoresis chamber, filled with electrophoresis buffer for alkaline comet assay $\left(1 \mathrm{mM} \mathrm{Na} \mathrm{N}_{2} \mathrm{EDTA} ; 300 \mathrm{mM}\right.$ $\mathrm{NaOH} ; \mathrm{pH}>13)$. After 40 min adaptation to the buffer, electrophoresis was performed $\left(25 \mathrm{~V} ; 300 \mathrm{~mA} ; 4^{\circ} \mathrm{C} ; 20 \mathrm{~min}\right)$. For neutralization, the slides were washed three times with Tris-buffer ( $400 \mathrm{mM}$ Tris; $\mathrm{pH} 7.4$ ) and dried at room temperature. Comets were visualized by ethidium bromide staining $(20 \mu \mathrm{g} / \mathrm{mL})$ and examined at 200 -fold magnification with a fluorescence microscope (Zeiss, Oberkochen Germany), using xenon lamp and ethidium bromide filter set (excitation at $\lambda=520 \mathrm{~nm}$ ). The images were recorded with a CCD Camera ('Xaw TV'). For a quantitative analysis of the DNA breaking, the tailmoment (amount of DNA damage) was evaluated using comet scoring software (http:// www.autocomet.com/home.php). The results are given as mean of tailmoment $\pm \operatorname{SEM}(n=4)$. At least 1000 cells/treatment were evaluated.

\section{Cell Proliferation on Laser-structured Spike Surfaces}

To test the influence of the material surface on cellular behavior, proliferation profiles of human fibroblasts and SH-SY5Y neuroblastoma cells were compared. After a cultivation time of $48 \mathrm{~h}$ the adherent cells 
were trypsinized. To determine the cell density, the cell suspension was collected and centrifuged at $800 \mathrm{~g}$ for $15 \mathrm{~min}$. The cells in pellet were resolved in cell culture medium and counted using a Rosenthal cell counter device. For a better comparison between the experiments, the cell densities were normalized on the seeding density at time $0 \mathrm{~h}$ (1.6 $10^{6}$ cell $\mathrm{s} / \mathrm{mL}$ ). The results are given as average (in percent) $\pm \mathrm{SEM}$ for $n=4$ experiments.

\section{Investigation of Cell Morphology and Adhesion Pattern}

The cell morphology of human fibroblasts and SH-SY5Y neuroblastoma cells was analyzed by fluorescence after nucleus and actin filaments staining using DAPI and phalloidin-Alexa 488, respectively (Molecular probes Invitrogen, Karlsruhe, Germany). After $24 \mathrm{~h}$ cultivation time, cells grown on the samples were fixed by a 10 min incubation in PBS containing 4\% formaldehyde. The cells were permeabilized by incubation in PBS containing $0.3 \%$ Triton X-100 for $10 \mathrm{~min}$. The chromatin in nucleus was stained by an incubation in PBS containing $1 \mu \mathrm{M}$ DAPI for $10 \mathrm{~min}$. After washing with PBS actin filaments were stained with $0.6 \mathrm{U}$ phalloidin-Alexa 488 solved in PBS for $1 \mathrm{~h}$ in the dark. For further analysis the cells were conserved in PBS.

The morphology of the cells was observed with a fluorescence microscope (Nikon TE 2000-E, Nikon, Düsseldorf, Germany) at excitation lights of $348 \mathrm{~nm}$ for DAPI and $488 \mathrm{~nm}$ for phalloidin-Alexa. Images were acquired using a CCD camera and software 'E Z-C1 3.5' (Nikon, Düsseldorf, Germany). The quantitative evaluation of the results was performed with ImageJ software. First, length and width of each single nucleus $\left(L_{\mathrm{n}}, W_{\mathrm{n}}\right)$ and each single cell $\left(L_{\mathrm{c}}, W_{\mathrm{c}}\right)$ were measured. By calculating the quotient $L_{\mathrm{n}} / W_{\mathrm{n}}$ and $L_{\mathrm{c}} / W_{\mathrm{c}}$ the nucleus and cell dilation were, respectively, estimated. Second, the number of cell extensions such as filopodia, lamellipodia, and retraction fibers which were defined as appendages that taper off to the surface and to neighboring cells was counted. The results are given as mean \pm SEM for four single measurements. About 100 cells per treatment were evaluated.

To analyze the adhesion patterns of fibroblasts and neuroblastoma cells the surface reflectance interference contrast (SRIC) technique was used. By reflecting light at the interface between cell and the cultivation surface, SRIC microscopy allows the visualization of focal contacts. The closer the adhesion contacts between the cell and the surface the darker appear interference fringes. SRIC analysis is limited to transparent surfaces, therefore, only glass samples were investigated. After $24 \mathrm{~h}$ cultivation time the cells were fixed with $4 \%$ formaldehyde solved in PBS 
for $10 \mathrm{~min}$ and conserved in PBS. Images were recorded using a fluorescence microscope equipped with a SRIC filter set (Nikon TE 2000-E, Nikon, Düsseldorf, Germany) and a CCD camera. The software 'NIS Elements AR 3.0' (Nikon, Düsseldorf, Germany) was used to acquire, conserve, and evaluate the images.

\section{RESULTS}

\section{Structure Fabrication and Surface Analysis}

In our experiments, we have controlled the surface topography parameters (spike dimensions and spike to spike distance) by adjusting the laser fluence. We found that by varying the laser fluence from 0.36 to $3.6 \mathrm{~J} / \mathrm{cm}^{2}$, the spike to spike distance changed from 2 to $15 \mu \mathrm{m}$, and the spike height from 1 to $20 \mu \mathrm{m}$ (Figure 1). Comparing the results of laser machined samples and unmachined reference samples, no differences in the EDX spectras could be observed. Therefore, one can consider that femtosecond laser machining of the silicon samples does not affect the material chemistry.
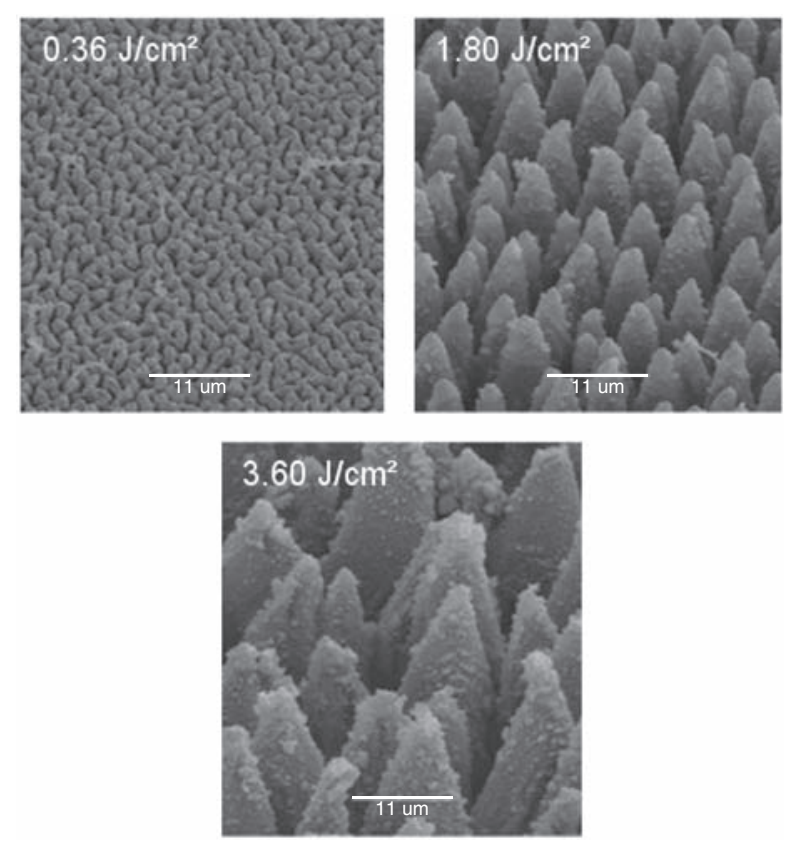

Figure 1. SEM images of spike structures fabricated on silicon (Si) at different laser fluences. 
Table 1. Contact angle results given as average \pm SEM of four independent measurements for each type of surface. Proliferation results of fibroblasts and neuroblastoma cells in dependence of materials after $48 \mathrm{~h}$ cultivation time. The results were normalized on cell density at time $0 \mathrm{~h}\left(1.610^{6} \mathrm{cel} / \mathrm{s} / \mathrm{mL}\right)$ and given as average in percentage \pm SEM of four independent measurements.

\begin{tabular}{lccc}
\hline & Contact & \multicolumn{2}{c}{ Proliferation (\%) \pm SEM } \\
\cline { 3 - 4 } & $\begin{array}{c}\text { angle }\left({ }^{\circ}\right) \\
\pm \text { SEM }\end{array}$ & Fibroblasts & $\begin{array}{c}\text { Neuroblastoma } \\
\text { cells }\end{array}$ \\
\hline Control & $72 \pm 3$ & $283.32 \pm 57.74$ & $230.86 \pm 47.23$ \\
Silicon (nonstructured) & $62 \pm 3$ & $290.36 \pm 22.08$ & $257.12 \pm 52.20$ \\
Silicon spikes & $130 \pm 3$ & $155.52 \pm 14.90$ & $251.62 \pm 28.05$ \\
Silicone elastomer (nonstructured) & $110 \pm 3$ & $224.01 \pm 8.96$ & $243.42 \pm 26.03$ \\
Silicone elastomer spikes replica & $159 \pm 3$ & $95.94 \pm 12.15^{\star}$ & $258.91 \pm 28.76$ \\
\hline
\end{tabular}

*In comparison to cultivation on control surface, the effect is statistically significant $(p<0.05$ Student's $t$-test analysis).

(a)

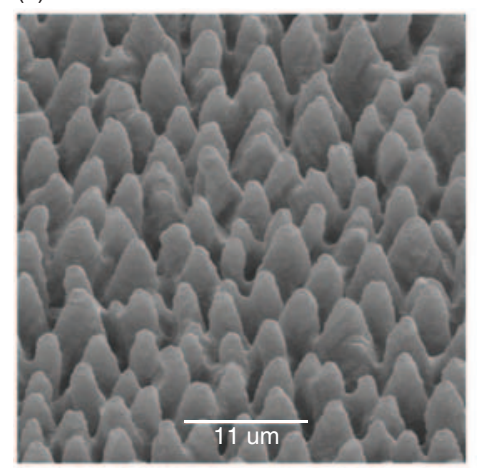

(b)

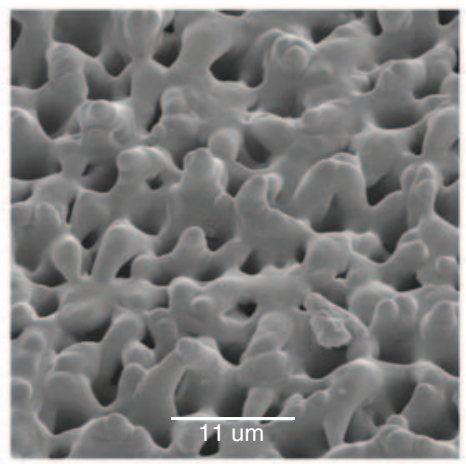

Figure 2. SEM images of Si spikes structures (a) and showing a negative relief structure in silicone fabricated by replication of Si spike structures (b).

It has been previously reported that structuring of a silicon surface with spikes enables tuning of wetting properties [19]. Indeed, we observed that the water contact angle varied with increased laser fluence from $62^{\circ}$ (reference sample, flat silicon) up to $130^{\circ}$ (spike structures) (Table 1). This increase of the contact angle was only due to the changed topography, as no chemical changes could be detected.

For the cell experiments, we fabricated spike structures with an average height of $5.9 \mu \mathrm{m}$, and a spike-to-spike distance of $4.8 \mu \mathrm{m}$ (in the following called cell test spike structures). The average top flattening of the spikes was $1.4 \mu \mathrm{m}$ in diameter (Figure 2). These spike dimensions are smaller than the size of the cells used in our experiments. 
The cell test spike structures were replicated in silicone elastomer MED-4234. The SEM-images of the inverse spike structures in silicone showed a good reproducibility of this method and a high-quality of manufactured topography even when replicated many times.

The characterization of the wettability of the inverted spikes revealed that these structures changed the water contact angle value from $110^{\circ}$ (flat silicone surface) to $159^{\circ}$ (Table 1 ).

\section{Material Genotoxicity Correlated with Material Chemistry}

Genotoxicity can be investigated by the comet assay method which detects single and double DNA strand breaks via a single-cellgelelectrophoresis. These DNA damage effects can quantitatively be described with the parameter tailmoment that characterizes the amount of damaged DNA. By comparing human fibroblasts and SH-SY5Y neuroblastoma cells cultivated on nonstructured and structured spike surfaces, it could be shown that both nonstructured materials did not significantly increase the incidence of DNA damages. As seen in Table 2, the tailmoments of Silicon (Si) spikes and silicone elastomer spikes replica were comparable with the control (for fibroblasts $1.48 \pm 0.13$, for neuroblastoma cells $1.25 \pm 0.1$ ). Only silicone elastomer spikes increased DNA damages for both cell types significantly ( $p<0.05$ Student's $t$-test), the tailmoments to $2.57 \pm 0.52$ for fibroblasts and $1.86 \pm 0.18$ for neuroblastoma cells. For this reason these samples were not used in further experiments.

Table 2. Analysis of material genotoxicity of fibroblasts and neuroblastoma cells demonstrated by comet assay. The results are given as average of tailmoment as a marker of DNA damages \pm SEM for four independent experiments. At least 1000 cells per treatment were evaluated.

\begin{tabular}{lcc}
\hline & \multicolumn{2}{c}{ Tailmoment \pm SEM } \\
\cline { 2 - 3 } & Fibroblasts & $\begin{array}{c}\text { Neuroblastoma } \\
\text { cells }\end{array}$ \\
\hline Control & $1.48 \pm 0.13$ & $1.25 \pm 0.1$ \\
Silicon (nonstructured) & $1.4 \pm 0.34$ & $1.24 \pm 0.19$ \\
Silicon spikes & $1.48 \pm 0.52$ & $1.09 \pm 0.17$ \\
Silicone elastomer (non-structured) & $1.53 \pm 0.5$ & $1.13 \pm 0.28$ \\
Silicone elastomer spikes & $2.57 \pm 0.52^{\star}$ & $1.86 \pm 0.18^{\star}$ \\
Silicone elastomer spikes replica & $1.3 \pm 0.33$ & $1.45 \pm 0.25$ \\
\hline
\end{tabular}

*In comparison to cultivation on control surface, the effect is statistical significant $(p<0.05$ Student's $t$-test analysis). 


\section{Laser-fabricated Spike Structures Affected Cell Proliferation}

In order to test the influence of the material surface topography on cellular behavior, proliferation profiles of human fibroblasts and SHSY5Y neuroblastoma cells grown on the femtosecond laser fabricated silicon (Si) spikes as well as on the silicone elastomer negative replicas were studied after $48 \mathrm{~h}$ cultivation. Nonstructured samples and glass slides served as references. For neuroblastoma cells we observed that none of the tested silicon ( $\mathrm{Si}$ ) and silicone elastomer samples (non- and microstructured) significantly affected the cell proliferation. Cultivated on the spike structures, the neuroblastoma cells proliferated at the same rate as those cultivated under control conditions on glass slides and achieved around $230.86 \% \pm 47.23$ to $258.91 \% \pm 28.76$ after $48 \mathrm{~h}$ cultivation. For the fibroblasts a tendency to reduction of proliferation on silicon ( $\mathrm{Si}$ ) spikes and silicone elastomer was observed. While the fibroblasts proliferated to $220-290 \%$ on control glass, nonstructured silicon or nonstructured silicone elastomer, they showed a reduction of proliferation to $155.52 \% \pm 14.9$ and $95.94 \% \pm 12.15$ on silicon-spikes and on spike replicas in silicone elastomer (Table 1). On the spike replicas in silicone elastomer, the reduction of proliferation was statistical significant ( $p<0.05$ Student's $t$-test) compared to the control glass surface.

\section{Spike Structures Changed the Cell Morphology}

Biomaterial-cell interactions can also be characterized by material effects on cell morphology. For this reason the nuclei and actin filaments of human fibroblasts and SH-SY5Y neuroblastoma cells cultivated on nonstructured and structured silicon (Si) samples were stained and the dimensions were quantified (Figure 3). By measuring the length and width of the nuclei, it was observed that the ratio between the length and the width $\left(L_{\mathrm{n}} / W_{\mathrm{n}}\right)$ was not affected by different cultivation surfaces and surface topology. On the control and silicon (Si) spike structures fibroblasts showed a ratio of $1.59 \pm 0.04$ and $1.97 \pm 0.11$, neuroblastoma cells a ratio of $2.34 \pm 0.09$ and $1.48 \pm 0.04$, respectively. On the contrary the cell dilation defined as the ratio between cell length and width $\left(L_{\mathrm{c}} / W_{\mathrm{c}}\right)$ was affected in both cell types. Fibroblasts and neuroblastoma cells presented a more round shape. For fibroblasts a $L_{\mathrm{c}} / W_{\mathrm{c}}$ of $6.74 \pm 0.41$ and $2.92 \pm 0.26$ was found for cells cultivated on glass and on the silicon ( $\mathrm{Si}$ ) spikes, respectively. For neuroblastoma cells the values of $L_{\mathrm{c}} / W_{\mathrm{c}}$ were $5.7 \pm 0.39$ (control) and $2.96 \pm 0.24$ (silicon ( $\mathrm{Si}$ ) spikes) (Figure 3(a)). Moreover, the average number of extensions such as filopodia, lamellipodia, and retraction fibers was evaluated. It was 

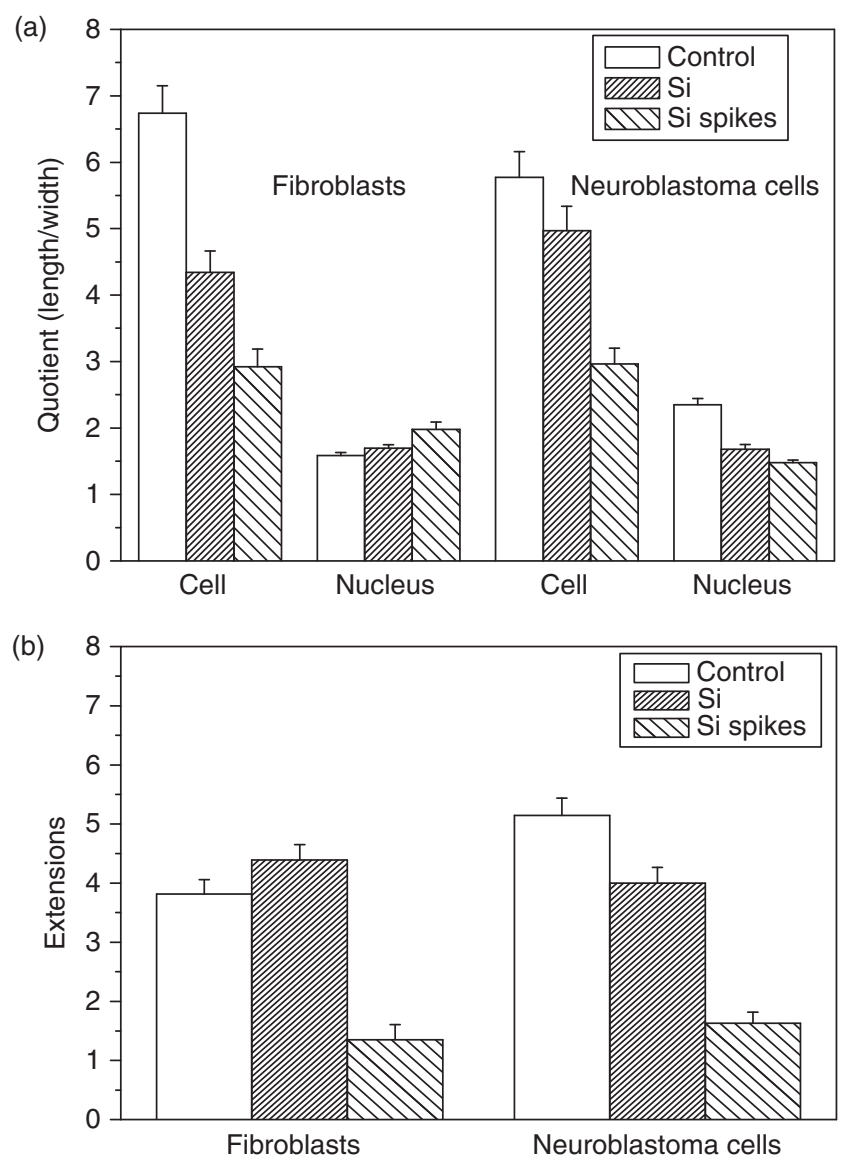

Figure 3. Analysis of cell morphology on silicon and silicone spikes after fluorescence staining of the nucleus and actin filaments. (a) Quantification of cell and nucleus size of fibroblasts and neuroblastoma cells and (b) number of extensions. The results are given as average \pm SEM of four single experiments. At least 100 cells per treatment were evaluated.

shown that nonstructured silicon ( $\mathrm{Si}$ ) did not have any effect on the formation of extensions, whereas the cells formed less extensions on the spike structures (for fibroblasts from $3.82 \pm 0.24$ on the control to $1.35 \pm 0.26$ on the silicon ( $\mathrm{Si}$ ) spikes; for neuroblastoma cells from $5.14 \pm 0.29$ to $1.63 \pm 0.18$, respectively). (Figure $3(\mathrm{~b})$ )

As the SRIC technique can only be performed on transparent materials, only the control adhesion patterns of human fibroblasts and SH-SY5Y neuroblastoma cells were examined. Figure 4 reveals that both cell types present differences in their adhesion patterns. The fibroblasts 
(a)

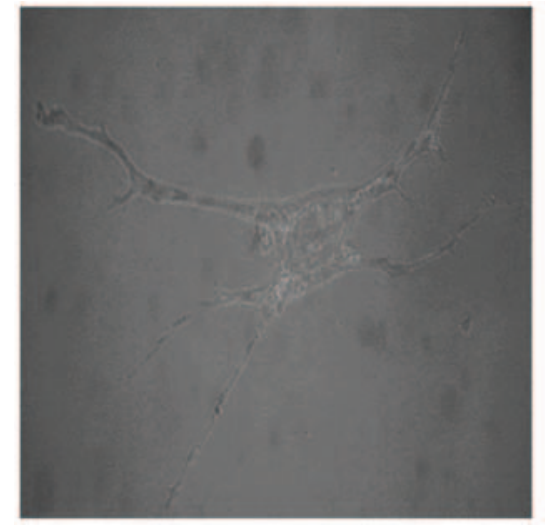

(b)

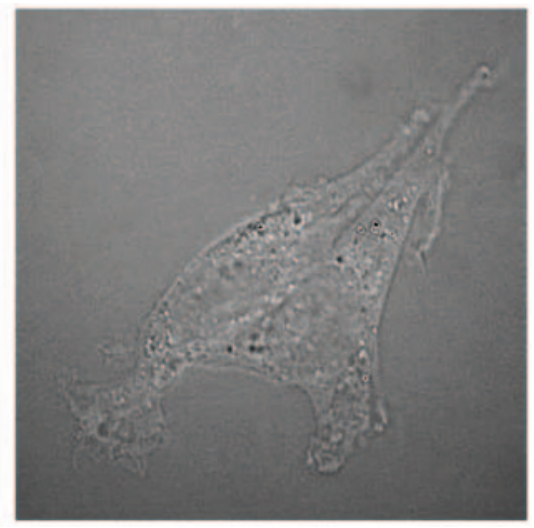

Figure 4. Adhesion pattern of human fibroblasts (a) and SH-SY5Y neuroblastoma cells (b) on control surfaces.

seemed to adhere with their whole cell body to the surface, while neuroblastoma cells formed many small focal contacts.

\section{DISCUSSION}

In the field of biomedicine and tissue engineering the knowledge about how cells interact with biomaterial surfaces is of great interest. Finding a biomaterial surface that controls and guides cellular behavior in a cell specific way would provide many biomedical applications and improve common implants. For this reason, the functionalization of biomaterial surfaces is a promising approach to achieve this goal. Functionalization can be achieved by changes in material chemistry, by material combination with bioactive molecules such as growth factors or by changes in surface topography. In all cases these material properties have to be reproducible and shall be stable under in vivo conditions.

Structuring with femtosecond laser provides reproducible surface topography with high precision nearly without material damaging. Apart from user defined surface designs femtosecond laser enables structuring with self-organized spike structures [15,17], ripple structures [16], a large variety of random nano- and microstructures [16].

In this article, the potential of quasi-periodical spikes structures for surface functionalization for biomedical applications is studied. These structures can be fabricated on silicon surfaces by irradiation with several hundred femtosecond laser pulses in a reactive gas atmosphere $\left(\mathrm{SF}_{6}\right)$. Thereby first (after irradiation with first laser pulses) a ripple 
structure with periodicity of the laser wavelength develops, subsequently with increasing number of laser pulses this pattern is replaced by bead structure. Finally, material is primarily ablated on the sides of the beads, forming spikes. Details of the formation procedure can be found in [17]. This fabrication process enables a tuning of geometrical structure size (height, density) in micrometer scale by varying of the laser fluence in a controlled and reproducible manner (Figure 1). Processes responsible for the transition from ripples to beads and establishing of the final surface topography are not yet fully understood. Tull et al. [17] have shown that the developing of different sized beads could be attributed to instabilities in the melted region, and a nonuniform melting temperature profile of the surface.

Spike structures can be transferred into different biocompatible materials by molding processes [20] or they can be directly produced in different important biocompatible metal such as titanium [15].

In our study we also investigated the potential of using spike structures as master structures for molding. We found that silicon spikes can be applied for rapid structuring of silicone elastomer (Figure 2), a widely used biocompatible material.

Topographical effects on cellular behavior were investigated with focus on genotoxicity, cell proliferation, and cell morphology. These studies were performed with human fibroblasts and SH-SY5Y neuroblastoma cells in order to determine cell specific responses. Structured silicone elastomer by direct laser ablation showed a genotoxic effect in both cell type (Table 2). We assume that laser radiation breaks the long polymer chains forming free radicals, which are responsible for the effect on the DNA strand breaks and other toxicity effects which we did not follow. Silicon $(\mathrm{Si})$ spikes and their replicas in silicone elastomer did not increase the incidences of DNA damages. Moreover, we observed that none of the tested silicon (Si) and silicone elastomer samples (non- and microstructured) significantly affected the proliferation of the neuroblastoma cells. These cells proliferated at the same rate as those cultivated under control conditions. On the contrary, for the fibroblasts a tendency to reduction of proliferation on silicon $(\mathrm{Si})$ spikes and on their replicas in silicone elastomer was observed (Table 1). On the spike replicas in silicone elastomer, the reduction of proliferation was significant compared to the control. At the morphological level, differences were seen for both cell types cultivated on nonstructured and spike structured silicon ( $\mathrm{Si}$ ) surfaces. In quantitative terms this is reflected by more roundness of the cells and less numbers of extensions (Figure 3). Hence, the morphology of the nuclei was not changed, in this case roundness of the cells does not correlate with either an increase of 
mitotic phases or an increase of cell death detectable through chromatin condensation. Comparable topographical effects on cell proliferation and cell morphology have already been reported in many other studies. Cell specific responses to defined surface structures as shown in this work are hardly described in literature. However, a detailed explanation for these effects still remains unclear. [9,10,21,22]

We can speculate that material effects on cellular behavior are basically caused by influences on cellular adhesion, which is considered to be the first step regarding surface-cell interactions. According to Adams [23] extensions play an important role in cell adhesion since they interact with components of the extracellular matrix. As the number of extensions was reduced for both cell types (Figure 3), it can be concluded that the spike structures affect the adhesion. Several studies show that proliferation, organization of the cytoskeleton, differentiation and so on only occur when the cells adhere to the surface [6-8]. Adhesion is mediated by components of the extracellular matrix such as fibronectin, collagen, laminin, etc. which are considered as ligands for adhesion receptors in the cell membrane such as different types of integrins [23-25]. With respect to the results of this report it can be hypothesized that the adhesion mechanism of human fibroblasts and SH-SY5Y neuroblastoma cells are different. Two observations sustain the hypothesis. (i) The structured surfaces induced cell specific responses with respect to proliferation (Table 1) and morphology (Figure 3). (ii) Analysis of adhesion under control conditions revealed that SH-SY5Y neuroblastoma adhered on the surface at restrain points (Figure 4(a)), whereas fibroblasts adhered with its whole body (Figure 4(b)).

The question is how one can make an estimation of the suitability of different topographies for a selected biomaterial surface with the goal to control cell adhesion. In our study we used wettability measurements on manufactured topographies for preliminary choice of topography designs for cell adhesion control and to provide a feedback for further structure design optimizations. Contact angle measurements are a fast and simple method to characterize the complete or incomplete wetting of a surface, which either depends on an increased or a reduced solid surface for contact. Analysis of the wettability of microstructured surfaces revealed that structuring induced an increase in hydrophobic character of the materials (Table 1). In other words, it reduced the surface area for contact. Therefore, the spike structures present a reduced surface area available for cell adhesion, which might explain the selective control of cellular behavior. With respect to cell specific adhesion patterns as shown in Figure 4, which reflect cellular contacts to material surfaces, one can conclude that the spike structures do not offer enough contact 
area for fibroblasts to adhere and proliferate, whereas neuroblastoma cells which typically need only small focal contacts to a surface are not affected by the structures. However, for a detailed understanding of the correlation between surface topographies and selective cell control, further analysis shall focus on material effects on adhesion with respect to adhesion ligands such as fibronectin, collagen, and laminin, as well as to cell components of the adhesion receptors such as integrins, vinculin, and focal adhesion kinase.

\section{CONCLUSION}

The fabrication of femtosecond laser-induced microstructures offers a variety of reproducable and defined size dimensions in silicon ( $\mathrm{Si}$ ) and also in soft materials such as silicone elastomer, which can be produced by a negative replication process. Surface structuring correlated with an increase of hydrophobicity due to a reduced surface area. With focus on biomaterial-cell interactions it is shown that topographical effects on cellular behavior occur in a cell specific manner. Therefore, this microstructuring technology represents a promising method useable for many different biomedical applications.

\section{ACKNOWLEDGMENTS}

This work was supported by the Deutsche Forschungsgemeinschaft (DFG), SFB 599, 'Zukunftsfähige bioresorbierbare und permanente Implantate aus metallischen und keramischen Werkstoffen'.

\section{REFERENCES}

1. Barbucci, R., Lamponi, S., Magnani, A. and Pasqui, D. Micropatterned Surfaces for the Control of Endothelial Cell Behaviour, Biomol. Eng., 2002: 19: $161-170$.

2. Dalby, M.J., Giannaras, D., Riehle, M.O., Gadegaard, N., Affrossman, S. and Curtis, A.S.G. Rapid Fibroblast Adhesion to 27nm High Polymer Demixed Nano-topography, Biomaterials, 2004: 25: 77-83.

3. Brors, D., Aletsee, C., Schwager, K. et al. Interaction of Spiral Ganglion Neuron Processes with Alloplastic Materials In Vitro(1), Hearing Res., 2002: 167: $110-121$.

4. Darby, I.A. and Hewitson, T.D. Fibroblast Differentiation in Wound Healing and Fibrosis, Int. Rev. Cytol., 2007: 257: 143-179.

5. Shinohara, T., Bredberg, G. and Ulfendahl, M. et al. Neurotrophic Factor Intervention Restores Auditory Function in Deafened Animals, PNAS, 2002: 99: $1657-1660$. 
6. Clark, E. and Hynes, R.O. Ras Activation is Necessary for Integrin-Mediated Activation of Extracellular Signal-regulated Kinase 2 and Cytosolic Phospholipase $\mathrm{A}_{2}$ but not for Cytoskeletal Organization, J. Biol. Chem., 1996: 271: 14814-14818.

7. Giancotti, F.G. and Ruoslahti, E. Integrin Signalling, Science, 1999: 285: 1028-1032.

8. Gumbiner, B.M. Cell Adhesion: The Molecular Basis of Tissue Architecture and Morphogenesis, Cell, 1996: 84: 345-357.

9. Choi, C.-H., Hagvall, S.H., Wu, B.M., Dunn, J.C.Y., Beygui, R.E. and Kim, C.-J. Cell Interaction with Three-dimensional Sharp-tip Nanotopography, Biomaterials, 2007: 28: 1672-1679.

10. Yim, E.K.F., Reano, R.M., Pang, S.W., Yee, A.F., Chen, C.S. and Leong, K.W. Nanopattern-induced Changes in Morphology and Motility of Smooth Muscle Cells, Biomaterials, 2005: 26: 5405-5413.

11. Cassie, A.B.D. and Baxter, S. Wettability of Porous Surfaces, Trans. Faraday Soc., 1944: 40: 546-551.

12. Wenzel, R.N. Resistance of Solid Surfaces to Wetting by Water, Ind. Eng. Chem., 1936: 28: 988-994.

13. Koch, J., Korte, F., Fallnich, C., Ostendorf, A. and Chichkov, B.N. Directwrite Subwavelength Structuring with Femtosecond Laser Pulses, Optical Engineering, 2005: 44: 051103.

14. Chichkov, B.N., Momma, C., Nolte, S., von Alvensleben, F. and Tuennermann, A. Femtosecond, Picosecond and Nanosecond Laser Ablation of Solids, Appl. Phys. A., 1996: 63: 109-115.

15. Nayak, B.K., Gupta, M.C. and Kolasinski, K.W. Formation of Nano-textured Conical Microstructures in Titanium Metal Surface by Femtosecond Laser Irradiation, Appl. Phys. A., 2008: 90: 399-402.

16. Vorobyev, A.Y. and Guo, C. Femtosecond Laser Structuring of Titanium Implants, Appl. Surf. Sci., 2007: 253: 7272-7280.

17. Tull, B.R., Carey, J.E., Mazur, E., McDonald, J.P. and Yalisove, S.M. Silicon Surfaces Morphologies after Femtosecond Laser Irradiation, MRS Bullet, 2006: 31: 626-633.

18. Schlie, S., Ngezahayo, A. and Ovsianikov, A. et al. Three-dimensional Cell Growth on Structures Fabricated from ORMOCER ${ }^{\circledR}$ by Two-photon Polymerisation Technique, J. Biomater. Appl., 2007: 22: 275-287.

19. Zobra, V., Persano, L. and Pisignano, D. et al. Making Silicon Hydrophobic: Wettability Control by Two-lengthscale Simultaneous Patterning with Femtosecond Laser Irradiation, Nanotechnology, 2006: 17: 3234-3238.

20. Reinhardt, C., Passinger, S., Zobra, V., Chichkov, B.N. and Fotakis, C. Replica Molding of Picosecond Laser Fabricated Si Microstructures, Appl. Phys. A, 2007: 87: 673-677.

21. Berry, C.C., Dalby, M.J., McCloy, D. and Affrossmann, S. The Fibroblast Response to Tubes Exhibiting Internal Nanotopography, Biomaterials, 2005: 26: 4985-4992.

22. Flemming, R.G., Murphy, C.J., Abrams, G.A., Goodman, S.L. and Nealey, P.F. Effects of Synthetic Micro- and Nano-structured Surfaces on Cell Behaviour, Biomaterials, 1999: 20: 573-588. 
23. Adams, J.C. Cell-matrix Contact Structures, Cell. Mol. Life Sci., 2001: 58: 371-392.

24. Ivaska, J., Pallari, H.-M., Nevo, J. and Eriksson, J.E. Novel Functions of Vimentin in Cell Adhesion, Migration, and Signalling, Exp. Cell Res., 2007: 313: 2050-2062.

25. Tzu, J. and Marinkovich, M.P. Bridging Structure with Function: Structural, Regulatory, and Developmental Role of Laminins, IJBCB, 2008: 40: 199-214. 